\title{
Balanced Coupled-Resonator Bandpass Filters Using Multisection Resonators for Common-Mode Suppression and Stopband Extension
}

\author{
Chung-Hwa Wu, Student Member, IEEE, Chi-Hsueh Wang, Member, IEEE, and Chun Hsiung Chen, Fellow, IEEE
}

\begin{abstract}
Novel fourth-order balanced coupled-resonator bandpass filters are proposed using suitably designed half-wavelength $(\lambda / 2)$ multisection resonators for common-mode suppression. By properly designing the input/output $(\mathrm{I} / \mathrm{O})$ resonators associated with the filter composed of four bi-section resonators, a balanced filter with good common-mode suppression is realized, but its rejection bandwidth is rather limited. To widen the rejection bandwidth, the $\mathrm{I} / \mathrm{O}$ bi-section resonators are replaced by the tri-section ones so that a balanced filter with good common-mode suppression and wide rejection bandwidth may be realized by suitably arranging the composed bi-/tri- section resonators. Specifically, a stopband-extended balanced filter with good common-mode suppression $(>50 \mathrm{~dB})$ within the differential-mode passband is implemented and its stopbands are also extended up to $5 f_{0}^{d}$ with a rejection level of $30 \mathrm{~dB}$, where $f_{0}^{d}$ is the center frequency in differential-mode operation.
\end{abstract}

Index Terms-Balanced filter, common-mode suppression, coupled-resonator bandpass filter, half-wavelength $(\lambda / 2)$ resonator, stopband extension.

\section{INTRODUCTION}

B ALANCED circuits are essential in building a modern communication system. Recently, under the trend of system-on-chip, it requires the integration of RF and analog circuits onto the digital baseband processor, thus the problem of interference and crosstalk from substrate coupling between components is getting more and more serious. A fully balanced transceiver architecture [1] with differential operation such as the one illustrated in Fig. 1 shows higher immunity to the environmental noise when compared with the unbalanced topology with single-ended signaling. Among various basic components, low-noise amplifiers, power amplifiers, mixers, and voltage-controlled oscillators have rapidly been developed as balanced circuits over the past few years. To establish a balanced system, the development of balanced filters is also necessary.

A well-designed differential-to-differential balanced bandpass filter should exhibit the desired differential-mode frequency response and should also be capable of reducing the

Manuscript received January 24, 2007. This work was supported in part by the National Science Council of Taiwan under Grant NSC 95-2752-E-002-001-PAE and in part by National Taiwan University under Excellent Research Project NTU-ERP-95R0062-AE00-00.

The authors are with the Department of Electrical Engineering and Graduate Institute of Communication Engineering, National Taiwan University, Taipei, 106 Taiwan, R.O.C. (e-mail: chchen@ew.ee.ntu.edu.tw).

Digital Object Identifier 10.1109/TMTT.2007.901609

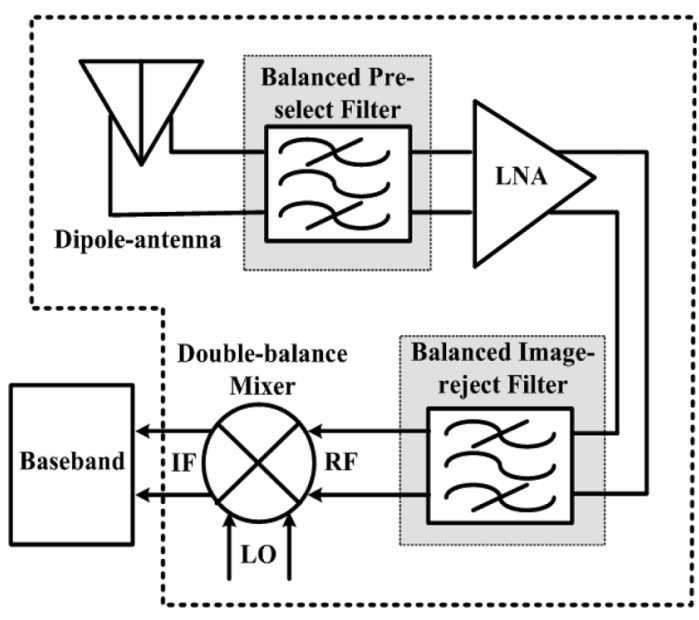

Fig. 1. Simplified architecture of the balanced transceiver.

common-mode signal, which is essential in increasing the signal-to-noise-ratio in the receiver and improving the efficiency of the dipole antenna in the transmitter. Furthermore, a well-designed balanced filter should also possess excellent out-of-band rejection and high selectivity. In particular, the wide-stopband bandpass filters are usually needed in conjunction with the nonlinear components (e.g., mixers or power amplifiers) so as to eliminate the undesired interference or noise in the stopband. However, pervious studies on the balanced filters with good common-mode suppression, high selectivity, and wide rejection bandwidth are rather limited [2]-[5].

Many single-ended filters with specified filtering characteristics have been developed in the past. Among them, parallel-coupled-line bandpass filters have the advantages of wide realizable bandwidth and simple synthesis procedures [6], [7]. Other popular ones are the coupled-resonator filters [8]-[10], particularly the narrowband bandpass filters that play a significant role in many applications. The coupled-resonator filters are implemented using the design technique based on the coupling coefficients and external quality factors, which may be applied to any type of resonator with different physical structures.

Recently, a fourth-order balanced coupled-line bandpass filter using parallel-coupled-line structures was proposed in [4], which presents high selectivity and good common-mode suppression within the passband. However, this filter has several drawbacks such as requiring a via-hole process and having a common-mode passband around $1.75 f_{0}^{d}$. To overcome the shortcomings in [4], another balanced filter was proposed in [5]. With the adoption of coupled stepped-impedance resonators, 
it is possible to extend the differential- and common-mode stopbands up to $5.5 f_{0}^{d}$ and also to avoid the undesired via-hole process. However, only the differential-mode quality factor is properly designed so that an acceptable common-mode rejection level of $34.46 \mathrm{~dB}$ is achieved around $f_{0}^{d}$.

In this study, two novel fourth-order balanced bandpass filters using $\lambda / 2$ multisection resonators are demonstrated to improve the common-mode suppression in [5]. In the first design, by properly designing the impedance ratio, length ratio, and tap position of the input/output (I/O) bi-section resonators, one may present the desired external quality factor in differentialmode operation and also give the complete reflection condition $\left(Z_{\text {in }}^{c}=0, Q_{e}^{c c}=\infty\right)$ in common-mode operation. Thus, a very low common-mode signal level may be achieved without any degradation of the insertion loss in differential-mode operation; however, its rejection bandwidth is still limited. Note that by using the bi-section resonators solely in the filter design, one may only achieve a balanced filter with either an extended stopband [5] or a good common-mode suppression, as demonstrated in this study, but not both. To realize a balanced filter with good common-mode suppression and stopband extension, both bi- and tri-section resonators are needed in the filter design. In the second design, to further extend the differential- and common-mode stopbands, the tri-section resonators are adopted for the I/O resonators. Specifically, by properly adjusting the I/O tri-section resonators, one may suitably misalign the corresponding differential- and common-mode higher order spurious resonance frequencies [11] so that a balanced filter with good common-mode suppression, as well as wider differential- and common-mode stopbands, may be realized.

\section{BALANCED FILTER USING BI-SECTION RESONATORS}

\section{A. Filter Structure}

The proposed fourth-order balanced filter shown in Fig. 2 is composed of four symmetric $\lambda / 2$ bi-section resonators. With the adoption of the symmetric structure, the proposed filter presents a perfect electric conductor wall under differential-mode excitation and a perfect magnetic conductor wall under commonmode excitation along the line of symmetry of the structure. Thus, it is possible to reduce the level of common-mode noise in addition to possessing the desired bandpass frequency response in differential-mode operation.

In this study, the $\lambda / 2$ bi-section resonator is adjusted for common-mode suppression, while in [5], it is used for stopband extension. Each $\lambda / 2$ bi-section resonator in Fig. 2, as illustrated by Fig. 3, is symmetric and has different characteristic impedances $Z_{i 1}, Z_{i 2}$ and lengths $L_{i 1}, L_{i 2}$, where the subscript $i$ denotes the resonators $a$ and $b$, respectively. The associated parameters such as the impedance ratio $R_{i}$ and the length ratio $\alpha_{i}$ are defined by

$$
\begin{aligned}
R_{i} & =\frac{Z_{i 2}}{Z_{i 1}}, \quad i=a, b \\
\alpha_{i} & =\frac{L_{i 2}}{\left(L_{i 1}+L_{i 2}\right)}, \quad i=a, b .
\end{aligned}
$$

Under differential-mode operation, a virtual short (perfect electric wall) would appear along the line of symmetry, therefore,

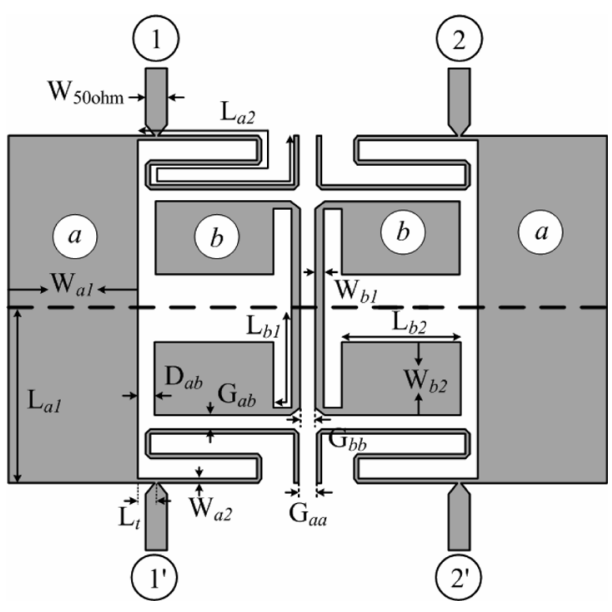

Fig. 2. Physical layout of the proposed fourth-order balanced filter using bi-section resonators $\left(W_{50} \Omega=1.9 \mathrm{~mm}, W_{a 1}=11.5 \mathrm{~mm}, W_{a 2}=0.4 \mathrm{~mm}\right.$, $W_{b 1}=0.8 \mathrm{~mm}, W_{b 2}=6.9 \mathrm{~mm}, L_{a 1}=15.5 \mathrm{~mm}, L_{a 2}=42 \mathrm{~mm}$, $L_{b 1}=11.9 \mathrm{~mm}, L_{b 2}=11.1 \mathrm{~mm}, L_{t}=2.2 \mathrm{~mm}, G_{a b}=0.5 \mathrm{~mm}$, $\left.G_{b b}=1.4 \mathrm{~mm}, G_{a a}=1.4 \mathrm{~mm}, D_{a b}=0.8 \mathrm{~mm}\right)$.

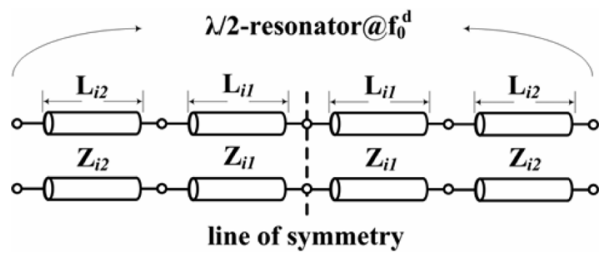

Fig. 3. Basic structure of $\lambda / 2$ bi-section resonator in Fig. 2.

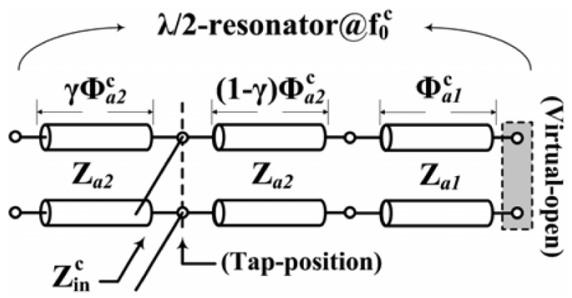

(a)

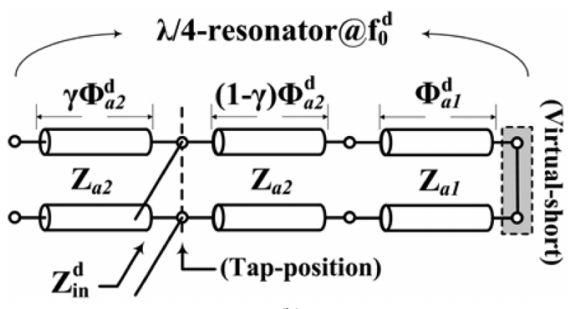

(b)

Fig. 4. Tapped I/O resonator (resonator a). (a) Common-mode equivalent half-circuit. (b) Differential-mode equivalent half-circuit. Here, $\phi_{a 1}^{c}=k \phi_{a 1}^{d}$, $\phi_{a 2}^{c}=k \phi_{a 2}^{d}$, and $k=f_{0}^{c} / f_{0}^{d}$.

each resonator, resonating at $f_{0}^{d}$, may be treated as a shorted quarter-wavelength $(\lambda / 4)$ resonator [see Fig. 4(b)]. Alternatively, under common-mode operation, a virtual-open (perfect magnetic wall) would be present along the line of symmetry, thus each resonator, now resonating at $f_{0}^{c}\left(f_{0}^{c}>f_{0}^{d}\right)$, may be regarded as a $\lambda / 2$ resonator with both ends opened [see Fig. 4(a)].

The proposed fourth-order balanced coupled-resonator bandpass filter in Fig. 2 is implemented in the microstrip structure 
on the FR4 substrate (substrate thickness $=1 \mathrm{~mm}$, dielectric constant $=4.3$, loss tangent $=0.02$, and metal thickness $=$ $0.03 \mathrm{~mm}$ ). In the filter design, the required coupling coefficients $M_{i j}$ and external quality factors $Q_{e}$ are extracted from the corresponding differential- and common-mode excitation circuits, which have the termination impedances $\left(2 Z_{o}\right)$ and $\left(Z_{o} / 2\right)$, respectively, as in [5]. In this study, the extract process is accomplished by the full-wave simulator ADS Momentum. As to the measurement, the balanced structure, as a four-port device, is first measured by the Agilent E5071B network analyzer to give the standard four-port $S$-parameters $S^{\text {std }}$. The two-port differential- and common-mode $S$-parameters, i.e., $S^{d d}$ and $S^{c c}$, may then be deduced from the four-port $S$-parameters $S^{\text {std }}$, as given in [4] and [12].

\section{B. Design Procedure}

The fourth-order balanced bandpass filter is designed to possess a quasi-elliptic response in the differential-mode operation with the center frequency at $f_{0}^{d}=1 \mathrm{GHz}$ and the fractional bandwidth $\Delta$ of $10 \%$. The corresponding element values of the low-pass prototype are $g_{1}=0.9545, g_{2}=1.3823$, $J_{1}=-0.1627$, and $J_{2}=1.06$. To determine the physical dimensions of the balanced filter, the differential-mode coupling coefficients $M_{i j}^{d d}$ and I/O external quality factors $Q_{e}^{d d}$ need to be calculated [13] as follows:

$$
\begin{aligned}
M_{a b}^{d d} & =\frac{\Delta}{\sqrt{g_{1} \cdot g_{2}}}=0.087 \\
M_{b b}^{d d} & =\frac{\Delta \cdot J_{2}}{g_{2}}=0.0767 \\
M_{a a}^{d d} & =\frac{\Delta \cdot J_{1}}{g_{1}}=-0.017 \\
Q_{e}^{d d} & =\frac{g_{0} \cdot g_{1}}{\Delta}=9.55 .
\end{aligned}
$$

To improve the common-mode suppression in addition to possessing the desired differential-mode bandpass response, the I/O resonators (resonator $a$ ) are both designed to possess the desired external quality factors $\left(Q_{e}^{d d}=9.55\right)$ in differential-mode operation and also to present a complete reflection $\left(Z_{\text {in }}^{c}=0\right.$, $\left.Q_{e}^{c c}=\infty\right)$ in common-mode operation. For this purpose, the impedance ratio, length ratio, and tap position of the I/O resonators should properly be arranged.

Fig. 4(a) and (b) shows the corresponding common- and differential-mode equivalent half-circuits for the tapped I/O resonators (resonator $a$ ), in which $\left(\phi_{a 1}^{d}, \phi_{a 2}^{d}\right)$ and $\left(\phi_{a 1}^{c}, \phi_{a 2}^{c}\right)$ are the corresponding differential- and common-mode electrical lengths, and $\gamma$ is the parameter associated with the tap position. By letting the common-mode input impedance be equal to zero $\left(Z_{\mathrm{in}}^{\mathrm{c}}=0\right)$ in Fig. 4(a), one may obtain the condition of complete reflection in common-mode operation $\left(Q_{e}^{c c}=\infty\right)$, which may be expressed as

$$
R_{a} \cdot \tan \left[\left(\frac{1-\gamma}{\gamma}\right) \cdot \frac{\pi}{2}\right]=\cot \left[\gamma \cdot \frac{\pi}{2} \cdot \frac{\phi_{a 2}^{d}}{\phi_{a 1}^{d}}\right] .
$$

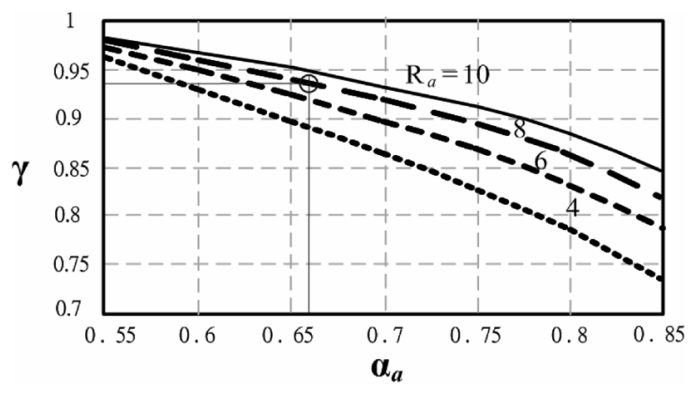

Fig. 5. Parameters to give complete reflection condition in common-mode operation.

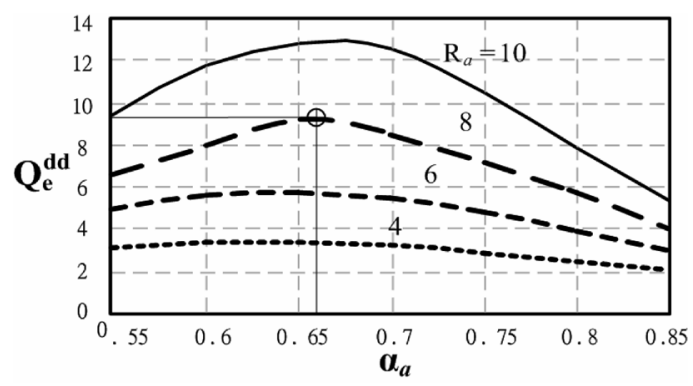

Fig. 6. Simulated $Q_{e}^{d d}$ versus the length ratio $\alpha_{a}$ under different values of impedance ratio $R_{a}$.

Additionally, for the differential-mode equivalent half-circuit of the I/O resonators [see Fig. 4(b)] to possess the differential-mode fundamental frequency at $f_{0}^{d}(=1 \mathrm{GHz})$, the resonance condition given by

$$
R_{a}=\tan \phi_{a 1}^{d} \cdot \tan \phi_{a 2}^{d}
$$

should also be satisfied. Thus, by combining (4) and (5), one may obtain the desired relation between the tap position parameter $\gamma$ and the length ratio $\alpha_{a}$ under different values of impedance ratio $R_{a}$, as depicted in Fig. 5, which is useful in designing the I/O bi-section resonator (resonator $a$ ) for good common-mode suppression.

Alternatively, the differential-mode external quality factor $Q_{e}^{d d}$ may be characterized by the ratio of $f_{0}^{d}$ and $\Delta f_{ \pm 90^{\circ}}^{d}$ [13], where $f_{0}^{d}$ is the center frequency in the phase response of $S_{11}^{d d}$ with reference to the differential-mode equivalent half-circuit shown in Fig. 4(b), and $\Delta f_{ \pm 90^{\circ}}^{d}$ should be determined from the frequencies at which the parameter $S_{11}^{d d}$ has a phase shift of $\pm 90^{\circ}$ with respect to the absolute phase at $f_{0}^{d}$. Hence, the corresponding differential-mode external quality factor $\left(Q_{e}^{d d}\right)$ is related to the differential-mode input impedance $\left(Z_{\text {in }}^{d}\right)$ and may be adjusted according to the given specification by properly choosing the values of impedance and length ratios $\left(R_{a}\right.$ and $\alpha_{a}$ ), as shown in Fig. 6. With the goal of giving $Q_{e}^{d d}=9.55$, the length ratio $\alpha_{a}=0.66$, impedance ratio $R_{a}=8$, and tap position parameter $\gamma=0.94$ are chosen as the initial values in determining the physical dimensions of the $\mathrm{I} / \mathrm{O}$ resonators ( $W_{a 1}, W_{a 2}, L_{a 1}$, and $\left.L_{a 2}\right)$ and the tap position $\left(L_{t}\right)$.

For easy comparison with [5], the physical dimensions of the inter-coupled resonators $\left(W_{b 1}, W_{b 2}, L_{b 1}\right.$, and $\left.L_{b 2}\right)$ are determined with the parameters $R_{b}=0.25$ and $\alpha_{b}=0.5$. Finally, 


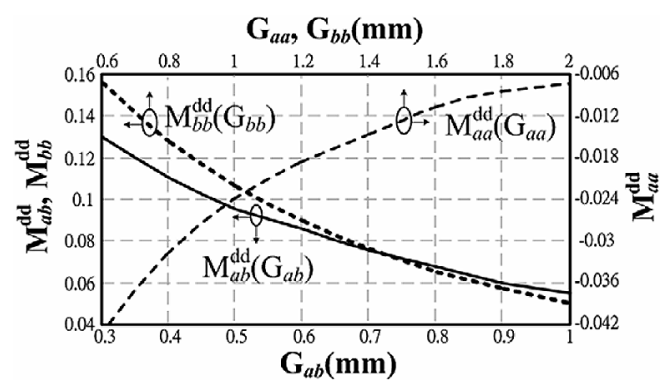

Fig. 7. Simulated differential-mode coupling coefficients versus the gaps between adjacent resonators.

the gaps $\left(G_{a b}, G_{a a}, G_{b b}\right.$, and $\left.D_{a b}\right)$ between adjacent resonators are obtained according to the corresponding differential-mode coupling coefficients $M_{i j}^{d d}$ in (3) $(i, j=a, b)$. The differential-mode coupling coefficients $M_{i j}^{d d}$ may be evaluated from the two split resonance frequencies associated with the coupled-resonator structure [13]. The design curves relating $M_{i j}^{d d}$ to the gaps between adjacent resonators are illustrated in Fig. 7. In the initial design phase, it is rather difficult to fully consider the discontinuity and coupling effects associated with the bi-section resonators. Thus, a fine-tune process based on full-wave simulation is needed to give the final physical dimensions, which are deviated approximately $10 \%$ from the initial values.

\section{Differential-Mode Response}

Fig. 8(a) and (b) shows the measured and simulated differential-mode frequency responses of the proposed balanced filter in Fig. 2. The measured differential-mode center frequency is at $1.025 \mathrm{GHz}$, the measured $3-\mathrm{dB}$ bandwidth is $10.5 \%$, and the minimum insertion loss is $4 \mathrm{~dB}$. This higher loss is mainly due to the use of a low-cost FR4 substrate, which has a high loss tangent of 0.02. The fabricated balanced filter is compact and has a size of $0.188 \lambda_{g} \times 0.323 \lambda_{g}(31 \mathrm{~mm} \times 53.26 \mathrm{~mm})$, where $\lambda_{g}$ is the guided wavelength of the microstrip structure at the differential-mode center frequency $\left(f_{0}^{d}=1 G H z\right)$. Fig. 8(b) shows the wideband differential-mode frequency responses ranging from 0.5 to $8.5 \mathrm{GHz}$. Note that this proposed balanced filter has only pushed the differential-mode stopband up to $3.1 \mathrm{GHz}\left(3 f_{0}^{d}\right)$ with a rejection level of $30 \mathrm{~dB}$. The implement filter has created two transmission zeros at 0.9 and $1.17 \mathrm{GHz}$, as expected. Moreover, an additional transmission zero is observed at $0.68 \mathrm{GHz}$, which is produced by the in-phase cancellation between two differential ports $\left(\angle S_{21}=\angle S_{2^{\prime} 1},\left|S_{21}\right|=\left|S_{2^{\prime} 1}\right|\right)$ and is not controllable.

Fig. 9(a) shows the distribution of differential-mode spurious resonance frequencies of each resonator up to $8.5 \mathrm{GHz}$. Note that the first and second spurious resonances of the I/O resonator (resonator $a$ ), which have been moved toward the center frequency $\left(f_{0}^{d}=1 \mathrm{GHz}\right)$, are now located at 2.315 and $3.16 \mathrm{GHz}$. Therefore, the differential-mode response may have a repeated passband around $3 \mathrm{GHz}\left(3 f_{0}^{d}\right)$.

\section{Common-Mode Response}

Fig. 8(a) and (c) shows the measured and simulated commonmode frequency responses of the proposed balanced filter in Fig. 2. The measured common-mode response is suppressed

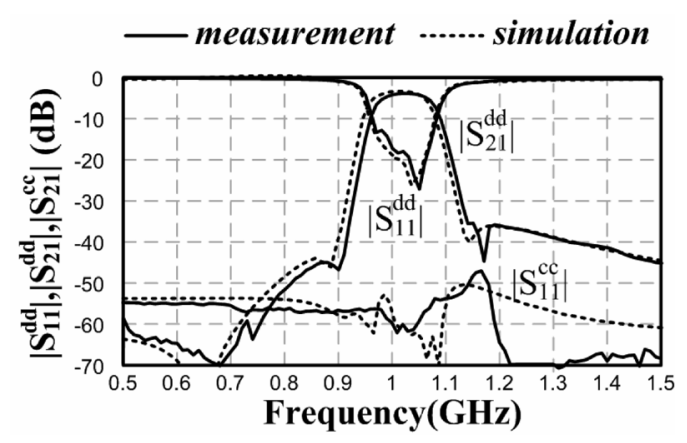

(a)

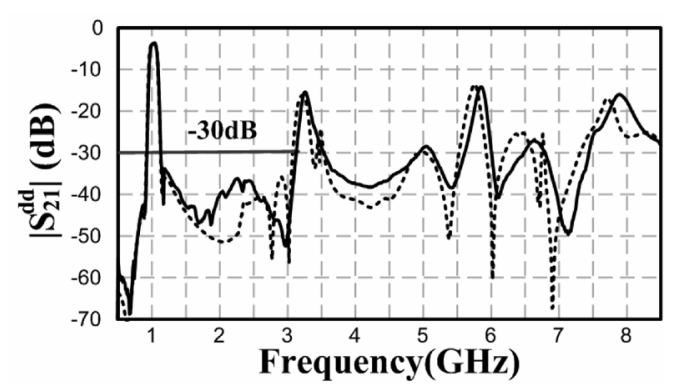

(b)

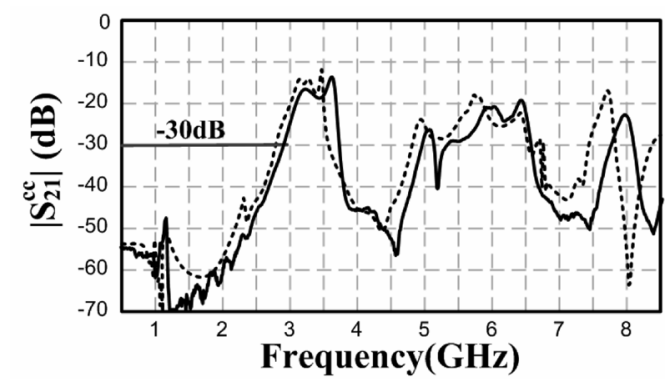

(c)

Fig. 8. Measured and simulated responses of the proposed balanced filter shown in Fig. 2. (a) Narrowband responses. (b) Wideband differential-mode responses. (c) Wideband common-mode responses.

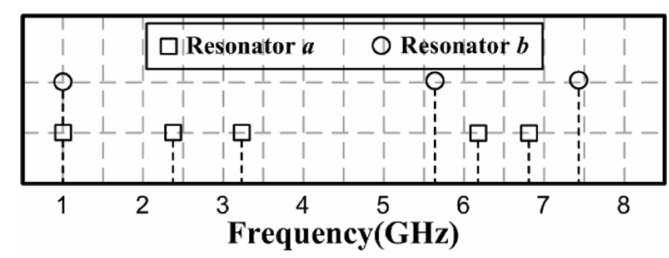

(a)

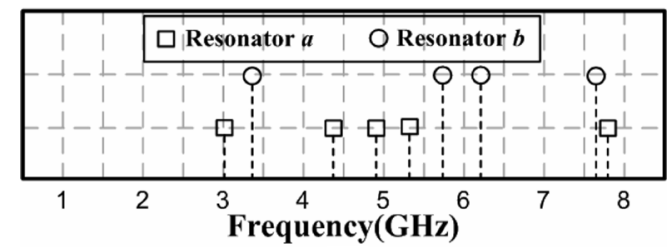

(b)

Fig. 9. Fundamental and harmonic frequencies of each resonator for the proposed balanced filter in Fig. 2. (a) Differential mode. (b) Common mode.

below $-54 \mathrm{~dB}$ within the passband. The proposed balanced filter shows a good common-mode signal suppression around $f_{0}^{d}$ $(1 \mathrm{GHz})$; however, it only pushes the common-mode stopband up to $2.9 \mathrm{GHz}\left(2.9 f_{0}^{d}\right)$ with a rejection level of $30 \mathrm{~dB}$. From 
the distribution of common-mode spurious resonance frequencies of each resonator, as shown in Fig. 9(b), one can find that the common-mode fundamental resonance frequencies of the resonators are located at 3 and $3.43 \mathrm{GHz}$, respectively. Therefore, the common-mode response has a passband around $3 \mathrm{GHz}$ $\left(3 f_{0}^{d}\right)$.

As suggested by [4], the common-mode rejection ratio (CMRR) defined by

$$
\mathrm{CMRR}=20 \log \frac{\left|S_{21}^{d d}\right|}{\left|S_{21}^{c c}\right|}(\mathrm{dB})
$$

is adopted as a figure-of-merit for qualitative characterizing the level of common-mode suppression around the passband of balanced filters. Specifically, the proposed balanced filter in Fig. 2 has a maximum CMRR of $58 \mathrm{~dB}$ at $f_{0}^{d}=1.025 \mathrm{GHz}$ with all values of CMRR above $54 \mathrm{~dB}$ from 0.97 to $1.08 \mathrm{GHz}$.

The measured results shown in Fig. 8 demonstrate that the design of I/O resonators to give proper external quality factors $\left(Q_{e}^{d d}=9.55\right.$ and $\left.Q_{e}^{c c}=\infty\right)$ is feasible to improve the commonmode suppression without degrading the differential-mode response. However, by the adoption of the bi-section resonators with impedance ratio $R_{a}=8\left(R_{a}>1\right)$, the higher order spurious resonance frequencies are moved toward the fundamental resonance frequency [14], [15]. Thus, the common-mode stopband of proposed balanced filter has only been pushed up to $2.9 \mathrm{GHz}\left(2.9 f_{0}^{d}\right)$, which is not satisfactory for modern wireless communication system.

\section{StOPBAND-EXTENDED BALANCED FiLTER USING BI-/TRI-SECTION RESONATORS}

\section{A. Filter Structure}

To further extend the stopbands of the proposed balanced filter in Fig. 2, the corresponding differential- and commonmode higher order resonance frequencies of the $\mathrm{I} / \mathrm{O}$ resonators should properly be arranged [11]. Here, by incorporating the $\lambda / 2$ tri-section resonators into the $\mathrm{I} / \mathrm{O}$ resonators, one may suitably separate the corresponding differential- and common-mode higher order resonance frequencies so that the stopbands may be further extended.

Shown in Fig. 10 is the proposed stopband-extended fourthorder balanced filter, which is composed of two $\lambda / 2$ bi-section resonators (resonator $b$ ) and two $\lambda / 2$ tri-section resonators (resonator $a$ ). Different from the one proposed in Fig. 2, the I/O resonators are replaced by the $\lambda / 2$ tri-section resonators. The $\lambda / 2$ tri-section resonator (resonator $a$ ) is symmetric and has different characteristic impedances $Z_{a 1}, Z_{a 2}$, and $Z_{a 3}$ and lengths $L_{a 1}$, $L_{a 2}$, and $L_{a 3}$, as illustrated in Fig. 11. Here, the lengths $L_{a 2}$ and $L_{a 3}$ are initially set identical $\left(L_{a 2}=L_{a 3}\right)$ in order to simplify the preliminary design. The associated parameters such as the impedance ratios $R_{a 2}$ and $R_{a 3}$ are defined by

$$
R_{a 2}=\frac{Z_{a 2}}{Z_{a 1}} \quad R_{a 3}=\frac{Z_{a 3}}{Z_{a 1}}
$$

and the length ratio $\alpha_{a}$ is again defined by (2). Note that the adoption of tri-section resonators is critical for stopbands extension; however, it also increases the complexity in the filter

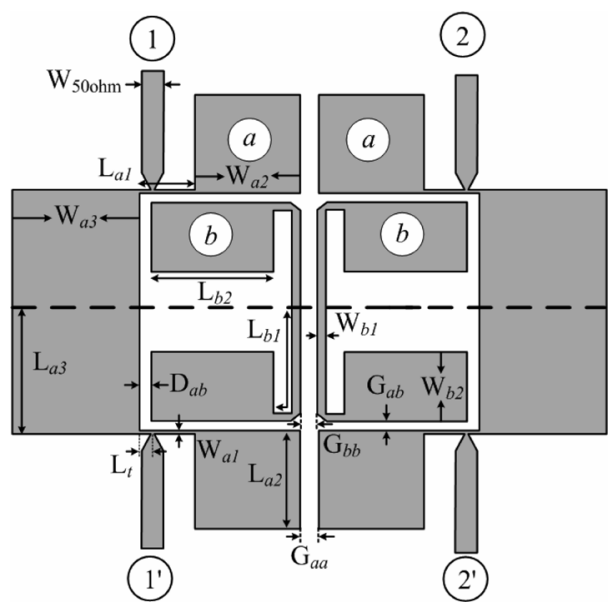

Fig. 10. Physical layout of the proposed stopband-extended fourth-order balanced filter using bi-/tri-section resonators $\left(W_{50 \Omega}=1.9 \mathrm{~mm}\right.$, $W_{a 1}=0.3 \mathrm{~mm}, W_{a 2}=9.3 \mathrm{~mm}, W_{a 3}=13 \mathrm{~mm}, W_{b 1}=0.8 \mathrm{~mm}$, $W_{b 2}=6.6 \mathrm{~mm}, L_{a 1}=4.9 \mathrm{~mm}, L_{a 2}=8.7 \mathrm{~mm}, L_{a 3}=10.8 \mathrm{~mm}$, $L_{b 1}=11.9 \mathrm{~mm}, L_{b 2}=11.3 \mathrm{~mm}, L_{t}=1.3 \mathrm{~mm}, G_{a b}=0.4 \mathrm{~mm}$, $\left.G_{b b}=1.4 \mathrm{~mm}, G_{a a}=1.6 \mathrm{~mm}, D_{a b}=0.5 \mathrm{~mm}\right)$.

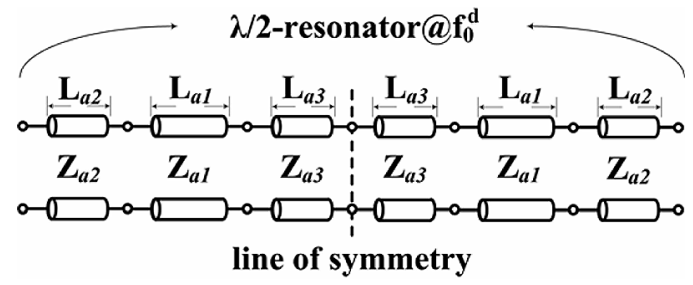

Fig. 11. Basic structure of the tri-section resonator (resonator $a$ ).

design. To simplify the idea, a proper approximation is introduced in the design phase.

\section{B. Design Procedure}

For simplicity, the lengths $L_{a 2}$ and $L_{a 3}$ of the tri-section resonators are assumed to be identical $\left(L_{a 2}=L_{a 3}\right)$ in the initial design phase, meanwhile, the width $W_{a 1}$ is set to be $0.3 \mathrm{~mm}$ $\left(Z_{a 1}=110 \Omega\right)$, which is the minimum width for fabrication. Therefore, only four variables $\left(W_{a 2}, W_{a 3}, L_{a 1}\right.$, and $\left.L_{a 2}\right)$ need to be determined. Fig. 12 shows the tapped structure for the $\mathrm{I} / \mathrm{O}$ resonators (resonator $a$ ). By letting the common-mode input impedance equal to zero $\left(Z_{\text {in }}^{c}=0, Q_{e}^{c c}=\infty\right)$ in Fig. 12(a), one may obtain the complete reflection condition in common-mode operation

$$
Z_{a 2} \cdot \tan \gamma \phi_{a 1}^{c}=Z_{a 3} \cdot \tan (1-\gamma) \phi_{a 1}^{c} .
$$

From (8), the impedance ratio $Z_{a 2} / Z_{a 3}$ versus $\phi_{a 1}^{c}$ under different values of $\gamma$ can be plotted as shown in Fig. 13, which is useful in designing the $\mathrm{I} / \mathrm{O}$ resonators for good common-mode suppression. Note that if the tap position parameter $\gamma=1 / 2$ is chosen, then $Z_{a 2} / Z_{a 3}=1$ is required to obtain $Q_{e}^{c c}=\infty$. When $\gamma=1 / 3$ or $\gamma=1 / 4$ is chosen, $Z_{a 2} / Z_{a 3}$ becomes a nonlinear function of $\phi_{a 1}^{c}$; however, it would nearly approach to a constant when $\phi_{a 1}^{c}$ is rather small (such as less than $40^{\circ}$ ). As a result, one may make the approximation that $Z_{a 2} / Z_{a 3}=2$ when $\gamma=1 / 3$ is selected, or $Z_{a 2} / Z_{a 3}=3$ when $\gamma=1 / 4$ is selected. Under such an approximation, one may provide a 


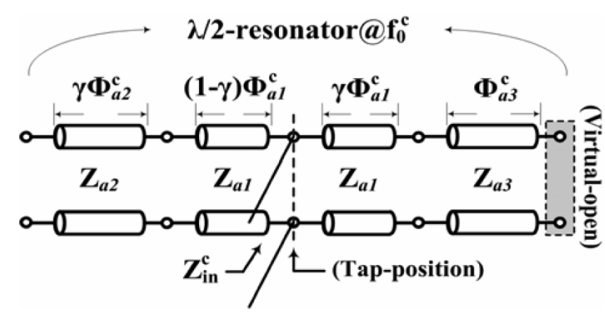

(a)

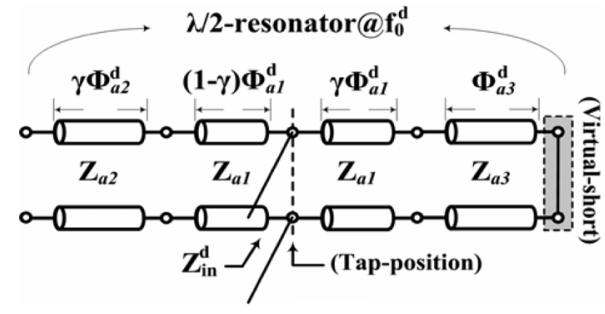

(b)

Fig. 12. Tapped I/O resonator (resonator $a$ ). (a) Common-mode equivalent half-circuit. (b) Differential-mode equivalent half-circuit.

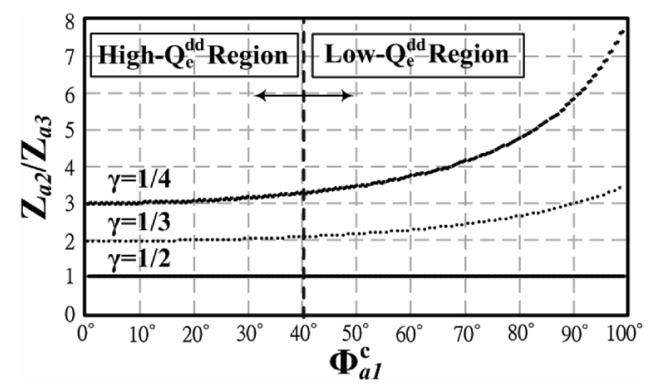

Fig. 13. Parameters to give complete reflection condition in common-mode operation.

large reflection $\left(Z_{\text {in }}^{c} \cong 0\right)$ in common-mode operation so that a very large $Q_{e}^{c c}$ may be achieved to suppress the common-mode signal.

Note that the above assumption and approximation would largely simplify the initial design procedure, especially in designing the narrowband filter, as discussed in this study, which requires $Q_{e}^{d d}=9.55$. Once the variable $\gamma$ and the impedance ratio $Z_{a 2} / Z_{a 3}$ are assigned, $Q_{e}^{c c}$ is almost fixed, and $Q_{e}^{d d}$ becomes a function of $\alpha_{a}, R_{a 2}$, and $R_{a 3}$, which can be adjusted according to the given specification. The simulated design curves for $Q_{e}^{d d}$ against the length ratio $\alpha_{a}$ under different values of $R_{a 2}$ and $R_{a 3}$ are plotted in Fig. 14(a) $(\gamma=1 / 2)$ and Fig. 14(b) $(\gamma=1 / 3)$. From Fig. 14(a) and (b), one may find that $Q_{e}^{d d}$ for the case $\gamma=1 / 2$ is too small to meet the specification $\left(Q_{e}^{d d}=9.55\right)$, thus, the case $\gamma=1 / 3$ is more suitable for realizing the desired. Based on the design curves in Fig. 14(b), the physical dimensions of I/O resonators $\left(W_{a 1}, W_{a 2}, W_{a 3}, L_{a 1}\right.$, and $\left.L_{a 2}\right)$ and the tap position $\left(L_{t}\right)$ may initially be determined. Here, the tap position parameter $\gamma=1 / 3$, length ratio $\alpha_{a}=0.8$, and impedance ratios $R_{a 2}=1 / 6$ and $R_{a 3}=1 / 12$ are chosen as the initial values.

The physical dimensions $\left(W_{b 1}, W_{b 2}, L_{b 1}\right.$, and $\left.L_{b 2}\right)$ of the inter-coupled resonators (resonator $b$ ) are then determined based on the chosen parameters $R_{b}=0.25$ and $\alpha_{b}=0.5$ as in the design of Fig. 2. Finally, the gaps $\left(G_{a b}, G_{a a}, G_{b b}\right.$, and $\left.D_{a b}\right)$

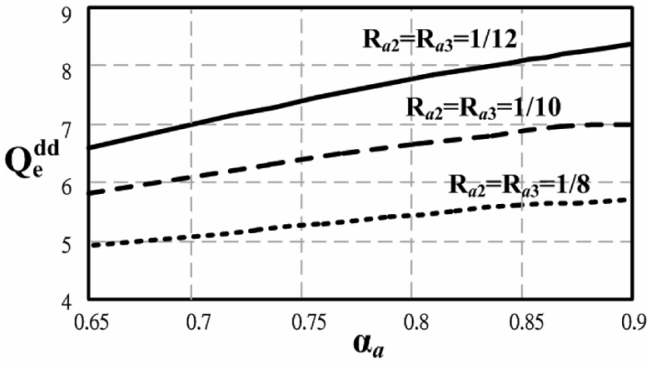

(a)

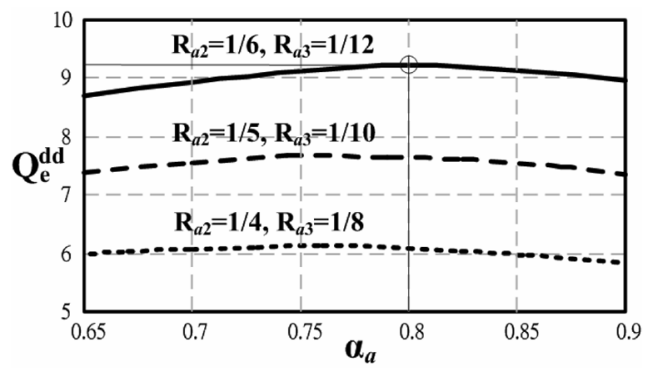

(b)

Fig. 14. Simulated $Q_{e}^{d d}$ versus the length ratio $\alpha_{a}$ under different values of impedance ratios $R_{a 2}$ and $R_{a 3}$. (a) $\gamma=1 / 2$. (b) $\gamma=1 / 3$.

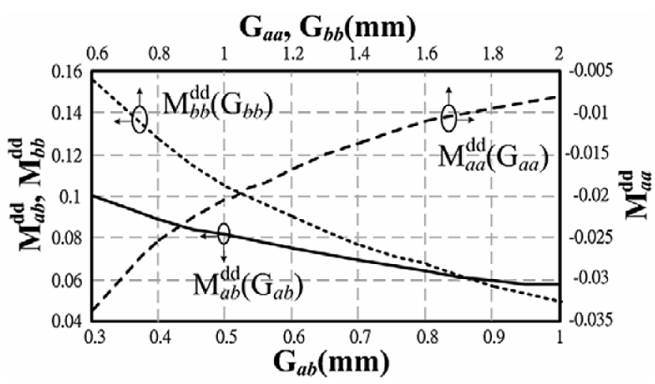

Fig. 15. Simulated differential-mode coupling coefficients versus the gaps between adjacent resonators.

between adjacent resonators are obtained according to the desired $M_{i j}^{d d}$ in (3). The design curves for $M_{i j}^{d d}$ relating to the gaps between adjacent resonators are illustrated in Fig. 15. Finally, a fine-tune process based on full-wave simulation is again required to relieve the assumption and approximation made in the simplified initial design and also to compensate for the discontinuity and coupling effects of the multisection resonators. Actually, this fine-tune process makes the final physical lengths $\left(L_{a 2}, L_{a 3}\right)$ not identical and causes a variation of almost $10 \%$ from the initial values.

\section{Differential-Mode Response}

Fig. 16(a) and (b) shows the measured and simulated differential-mode frequency responses of the proposed balanced filter in Fig. 10. The measured differential-mode center frequency is at $1.025 \mathrm{GHz}$, the measured $3-\mathrm{dB}$ bandwidth is $11.5 \%$, and the minimum insertion loss is $3.88 \mathrm{~dB}$ (again due to the high loss tangent of the FR4 substrate). The fabricated balanced filter has a size of $0.233 \lambda_{g} \times 0.34 \lambda_{g}(38.4 \mathrm{~mm} \times 56 \mathrm{~mm})$. Fig. 16(b) shows the wideband differential-mode frequency responses ranging from 0.5 to $8.5 \mathrm{GHz}$. From the distribution of differential-mode spurious resonance frequencies of each resonator, 


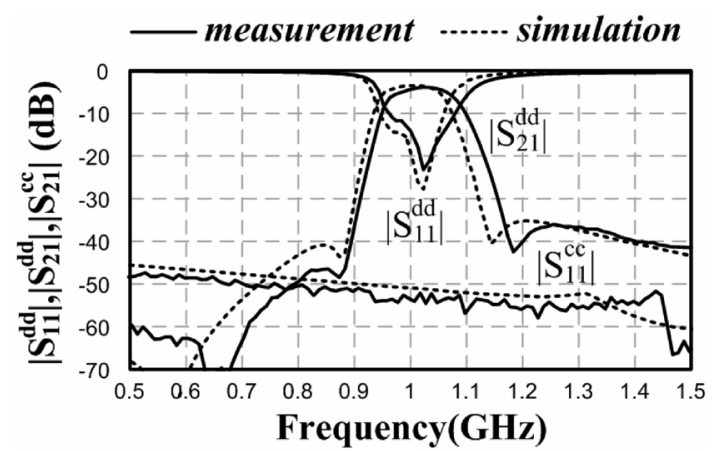

(a)

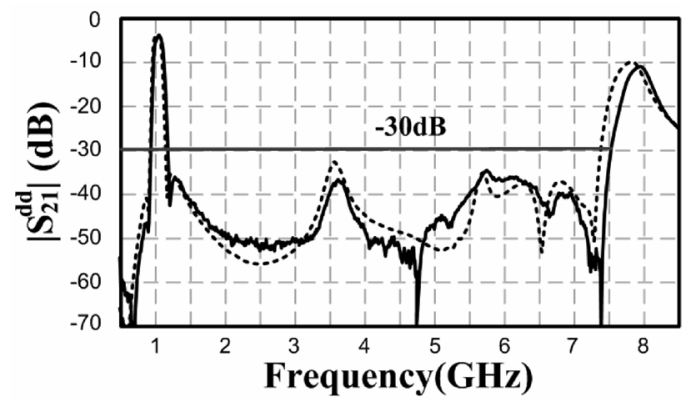

(b)

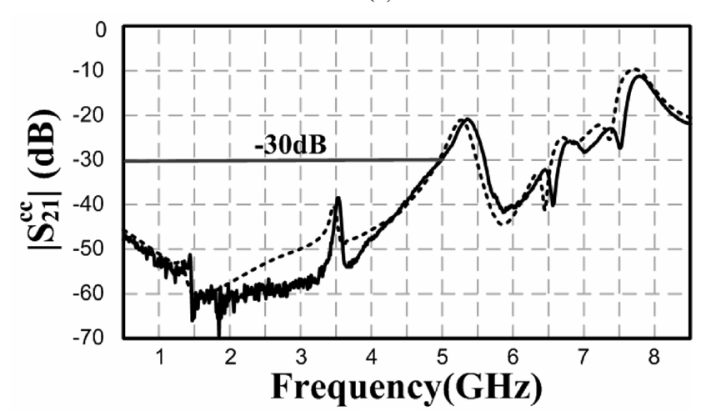

(c)

Fig. 16. Measured and simulated responses of the stopband-extended balanced filter shown in Fig. 10. (a) Narrowband responses. (b) Wideband differentialmode responses. (c) Wideband common-mode responses.

shown in Fig. 17(a), the differential-mode higher order spurious resonances have been separated from each other before 5.5 GHz. Therefore, the proposed stopband-extended balanced filter has a stopband up to which is almost twice wider than the one in Fig. 2. In addition to the preselected transmission zeros at 0.88 and $1.18 \mathrm{GHz}$, here an additional transmission zero is again produced at $0.68 \mathrm{GHz}$, a consequence of in-phase cancellation between two differential ports.

\section{Common-Mode Response}

Fig. 16(a) and (c) shows the measured and simulated common-mode frequency responses of the proposed balanced filter in Fig. 10. The measured common-mode response is suppressed below $-52.7 \mathrm{~dB}$ within the passband. Specifically, this proposed balanced filter shows good common-mode signal suppression around $f_{0}^{d}(1 \mathrm{GHz})$ and its common-mode stopband is pushed up to $5 \mathrm{GHz}\left(5 f_{0}^{d}\right)$ with a rejection level of $30 \mathrm{~dB}$. From the distribution of common-mode spurious resonance frequencies of each resonator, shown in Fig. 17(b), one can

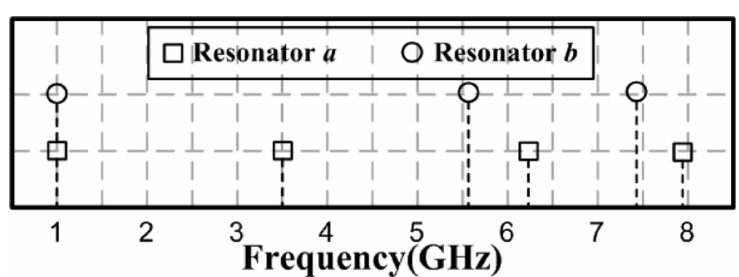

(a)

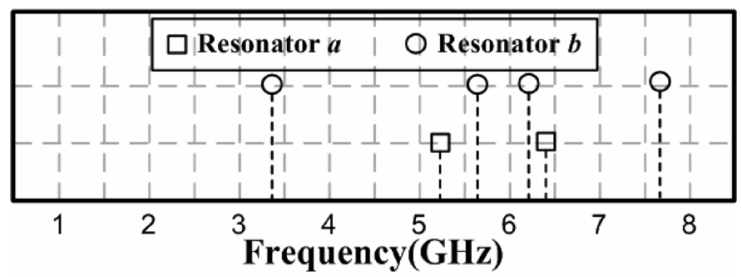

(b)

Fig. 17. Fundamental and harmonic frequencies of each resonator for the stopband-extended balanced filter in Fig. 10. (a) Differential mode. (b) Common mode.

TABLE I

PERFormanCE COMPARISON WiTh PREVIOUS STUDIES

\begin{tabular}{|c|c|c|c|c|c|}
\hline & $\begin{array}{c}f_{0}^{d} \\
(\mathrm{GHz})\end{array}$ & $\begin{array}{c}\text { Bandwidth } \\
(\%)\end{array}$ & $\begin{array}{c}\left|S_{21}^{\text {dd }}\right| @ f_{0}^{d} \\
(\mathrm{~dB})\end{array}$ & $\begin{array}{c}\text { Common-mode } \\
\text { rejection ratio } \\
\text { in passband (dB) }\end{array}$ & $\begin{array}{c}\text { Rejection } \\
\text { (below 30dB) } \\
\text { up to }\end{array}$ \\
\hline$[4]$ & 1.99 & 11 & 3.74 & $>40$ & $1.5 f_{0}^{d}$ \\
\hline$[5]$ & 1.02 & 12 & 3.51 & $>28$ & $5.5 f_{0}^{d}$ \\
\hline Fig. 2 & 1.025 & 10.5 & 4 & $>54$ & $2.9 f_{0}^{d}$ \\
\hline Fig. 10 & 1.025 & 11.5 & 3.88 & $>46.4$ & $5 f_{0}^{d}$ \\
\hline
\end{tabular}

find that the fundamental resonance frequencies are located at $3.5 \mathrm{GHz}$ (resonator $b$ ) and $5.2 \mathrm{GHz}$ (resonator $a$ ). However, the resonance at $5.2 \mathrm{GHz}$ would be the main factor in deciding the common-mode stopband response due to the stronger coupling between resonators $a$ [5].

The balanced filter in Fig. 10 has a maximum CMRR of $49.3 \mathrm{~dB}$ at $1.025 \mathrm{GHz}$ with all values of CMRR above $46.4 \mathrm{~dB}$ from 0.966 to $1.084 \mathrm{GHz}$. Note that the adoption of the I/O tri-section resonators not only provides a good common-mode suppression, but also possesses a higher flexibility in misaligning the differential- and common-mode spurious resonance frequencies so that the corresponding stopbands may also be extended.

Two balanced filters realized in this study are summarized and compared with the pervious study in Table I. Note that the proposed stopband-extended balanced filter depicted in Fig. 10 shows good CMRR of $46.4 \mathrm{~dB}$ within the passband and has a wider stopband up to $5 f_{0}^{d}$ with a rejection level of $30 \mathrm{~dB}$. Until now, the proposed stopband-extended balanced filter (Fig. 10) may be the one to give a better performance of good CMRR, high selectivity, and wide rejection bandwidth.

\section{CONCLUSION}

In this paper, two novel balanced filters using suitably designed I/O multisection resonators for common-mode 
suppression have been presented. Meanwhile, the design procedures and the corresponding differential- and common-mode frequency responses have also been carefully examined. By properly designing the I/O bi-section resonators associated with the filter composed of four bi-section resonators, it is possible to implement a balanced filter with good common-mode suppression; however, its rejection bandwidth is rather narrow (up to $2.9 f_{0}^{d}$ ). To realize a balanced filter with good common-mode suppression and wider rejection bandwidth, a novel filter structure composed of two I/O tri-section resonators and two inter-coupled bi-section resonators has been proposed. By properly designing the bi-/tri-section resonators, a stopband-extended balanced filter with good CMRR $(=46.4 \mathrm{~dB})$ within the passband has been implemented, and its stopband has been extended up to $5 f_{0}^{d}$ with a rejection level of $30 \mathrm{~dB}$.

\section{REFERENCES}

[1] C.-H. Wang, Y. H. Cho, C. S. Lin, H. Wang, C. H. Chen, D. C. Niu, J. Yeh, C. Y. Lee, and J. Chern, "A $60 \mathrm{GHz}$ transmitter with integrated antenna in $0.18 \mu \mathrm{m} \mathrm{SiGe} \mathrm{BiCMOS} \mathrm{technology,"} \mathrm{in} \mathrm{IEEE} \mathrm{Int.} \mathrm{Solid-}$ State Circuit Conf. Tech. Dig., Feb. 2006, pp. 186-187.

[2] A. Ziroff, M. Nalezinski, and W. Menzel, "A $40 \mathrm{GHz}$ LTCC receiver module using a novel submerged balancing filter structure," in Proc. Radio Wireless Conf., 2003, pp. 151-154.

[3] Y.-S. Lin and C. H. Chen, "Novel balanced microstrip coupled-line bandpass filters," in URSI Int. Electromagn. Theory Symp., 2004, pp. 567-569.

[4] C.-H. Wu, C.-H. Wang, and C. H. Chen, "Novel balanced coupled-line bandpass filters with common-mode noise suppression," IEEE Trans. Microw. Theory Tech., vol. 55, no. 2, pp. 287-295, Feb. 2007.

[5] C.-H. Wu, C.-H. Wang, and C. H. Chen, "Stopband-extended balanced bandpass filter using coupled stepped-impedance resonators," IEEE Microw. Wireless Compon. Lett., vol. 17, no. 7, Jul. 2007.

[6] S. B. Cohn, "Parallel-coupled transmission-line-resonator filters," IRE Trans. Microw. Theory Tech., vol. MTT-6, no. 7, pp. 223-231, Apr. 1958.

[7] C.-Y. Chang and T. Itoh, "A modified parallel-coupled filter structure that improves the upper stopband rejection and response symmetry," IEEE Trans. Microw. Theory Tech., vol. 39, no. 2, pp. 310-314, Feb. 1991.

[8] J. S. Hong and M. J. Lancaster, "Coupling of microstrip square open-loop resonator for cross-coupled planar microwave filters," IEEE Trans. Microw. Theory Tech., vol. 44, no. 12, pp. 2099-2109, Dec. 1996.

[9] S. Y. Lee and C. M. Tsai, "New cross-coupled filter design using improved hairpin resonators," IEEE Trans. Microw. Theory Tech., vol. 48, no. 12, pp. 2482-2490, Dec. 2000.

[10] C. C. Chen, Y. R. Chen, and C. Y. Chang, "Miniaturized microstrip cross-coupled filters using quarter-wave or quasi-quarter-wave resonators," IEEE Trans. Microw. Theory Tech., vol. 51, no. 1, pp. 120-131, Jan. 2003.

[11] S.-C. Lin, P.-H. Deng, Y.-S. Lin, C.-H. Wang, and C. H. Chen, "Wide-stopband microstrip bandpass filters using dissimilar quarter-wavelength stepped-impedance resonators," IEEE Trans. Microw. Theory Tech., vol. 54, no. 3, pp. 1011-1018, Mar. 2006.

[12] D. E. Bockelman and W. R. Eisenstant, "Combined differential and common-mode scattering parameters: Theory and simulation," IEEE Trans. Microw. Theory Tech., vol. 43, no. 7, pp. 1530-1539, Jul. 1995.
[13] J. S. Hong and M. J. Lancaster, Microstrip Filter for RF/Microwave Application. New York: Wiley, 2001.

[14] M. Makimoto and S. Yamashita, "Bandpass filters using parallel coupled stripline stepped impedance resonators," IEEE Trans. Microw. Theory Tech., vol. MTT-28, no. 12, pp. 1413-1417, Dec. 1980.

[15] M. Sagawa, M. Makimoto, and S. Yamashita, "Geometrical structures and fundamental characteristics of microwave stepped-impedance resonators," IEEE Trans. Microw. Theory Tech., vol. 45, no. 7, pp. 1078-1085, Jul. 1997.

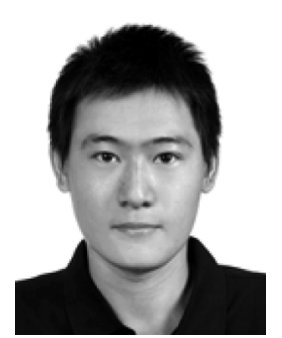

Chung-Hwa Wu (S'06) was born in Tainan, Taiwan, R.O.C., in 1982. He received the B.S. degree in electrical engineering from National Chung Hsing University, Taichung, Taiwan, R.O.C., in 2004, and is currently working toward the Ph.D. degree at the Graduate Institute of Communication Engineering, National Taiwan University, Taipei, Taiwan, R.O.C.

His research interests include the design and analysis of microwave filters and passive circuits.

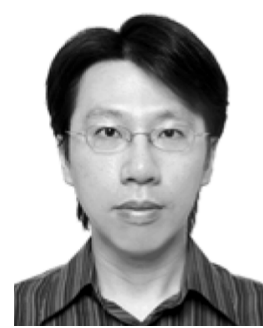

Chi-Hsueh Wang (S'02-M'05) was born in Kaohsiung, Taiwan, R.O.C., in 1976. He received the B.S. degrees in electrical engineering from National Cheng Kung University, Tainan, Taiwan, R.O.C., in 1997, and the Ph.D. degree from National Taiwan University, Taipei, Taiwan, R.O.C., in 2003.

$\mathrm{He}$ is currently a Post-Doctoral Research Fellow with the Graduate Institute of Communication Engineering, National Taiwan University. His research interests include the design and analysis of microwave and millimeter-wave circuits and computational elec-

tromagnetics.

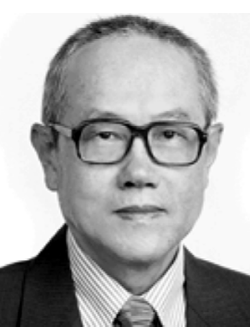

Chun Hsiung Chen (SM'88-F'96) was born in Taipei, Taiwan, R.O.C., on March 7, 1937. He received the B.S.E.E. and Ph.D. degrees in electrical engineering from National Taiwan University, Taipei, Taiwan, R.O.C., in 1960 and 1972, respectively, and the M.S.E.E. degree from National Chiao Tung University, Hsinchu, Taiwan, R.O.C., in 1962.

In 1963, he joined the Faculty of the Department of Electrical Engineering, National Taiwan University, where he is currently a Professor. From August 1982 to July 1985, he was Chairman of the Department of Electrical Engineering, National Taiwan University. From August 1992 to July 1996, he was the Director of the University Computer Center, National Taiwan University. In 1974, he was a Visiting Scholar with the Department of Electrical Engineering and Computer Sciences, University of California at Berkeley. From August 1986 to July 1987, he was a Visiting Professor with the Department of Electrical Engineering, University of Houston, Houston, TX. In 1989, 1990, and 1994, he visited the Microwave Department, Technical University of Munich, Munich, Germany, the Laboratoire d'Optique Electromagnetique, Faculte des Sciences et Techniques de Saint-Jerome, Universite d'Aix-Marseille III, Marseille, France, and the Department of Electrical Engineering, Michigan State University, East Lansing, respectively. His areas of interest include microwave circuits and computational electromagnetics. 